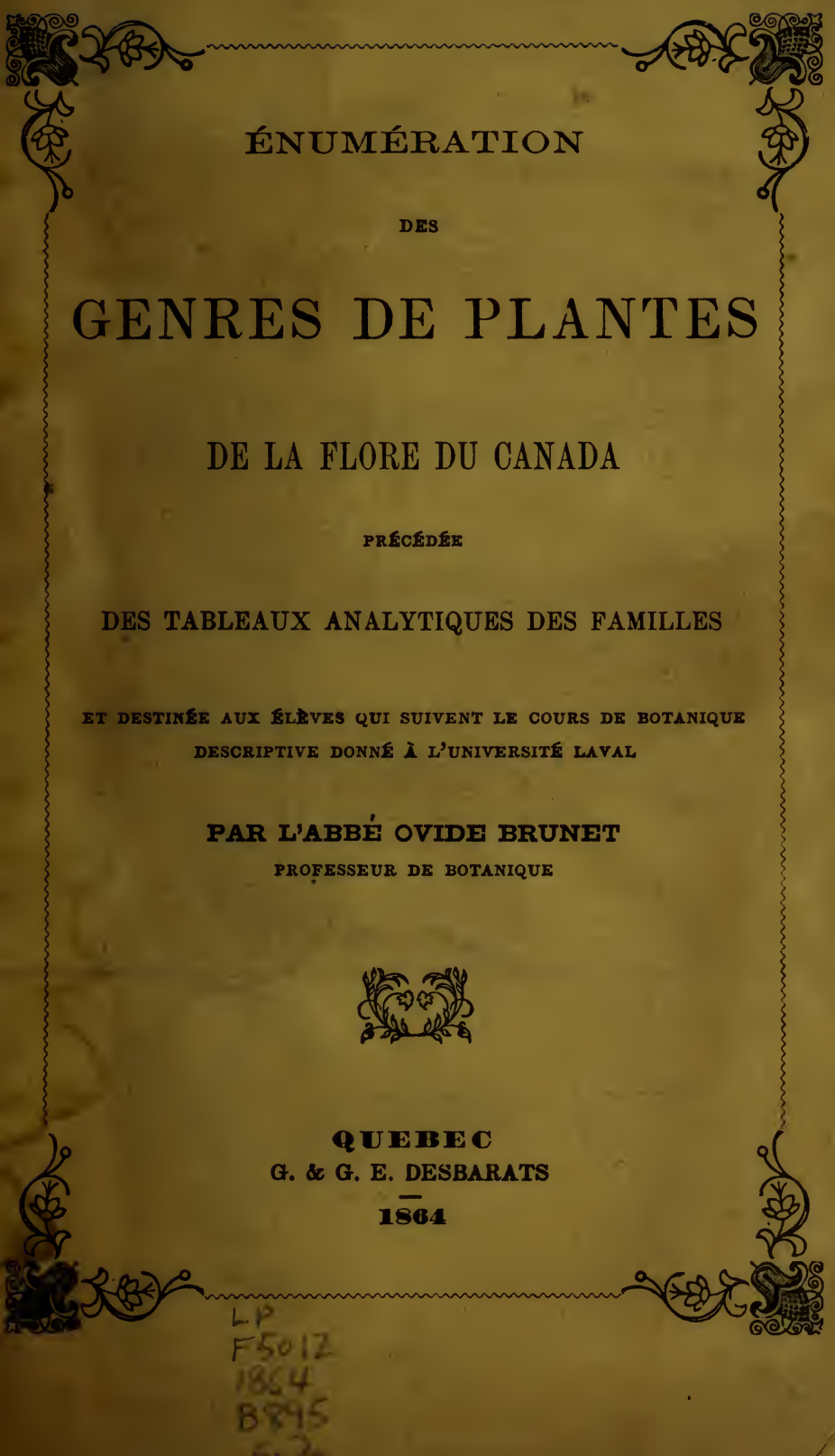




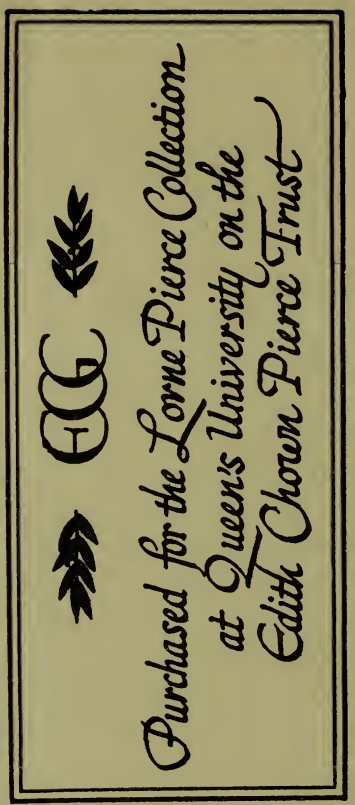




\title{
ÉNUMÉRATION
}

\author{
DES
}

\section{GENRES DE PLANTES}

\author{
DE LA FLORE DU CANADA
}

PRÉCEDEEE

DES TABLEAUX ANALYTIQUES DES FAMILLES

ET DESTINEE AUX ÉLÈVES QUI SUIVENT LE COURS DE BOTANIQUE DESCRIPTIVE DONNE À L'UNIVERSITÉ LAVAL

\section{PAR L'ABBÉ OVIDE BRUNET}

PROFESSEUR DE BOTANIQUE.

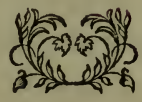

QUEDEC

G. \& G. E. DESBARATS

\section{$\overline{1864}$}




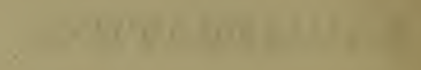

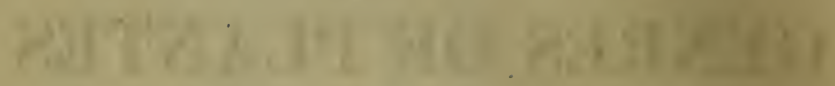

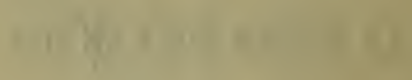

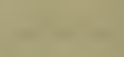

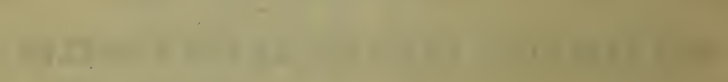

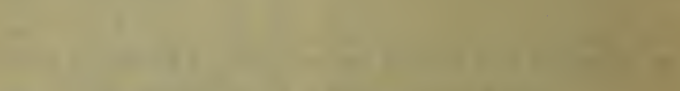

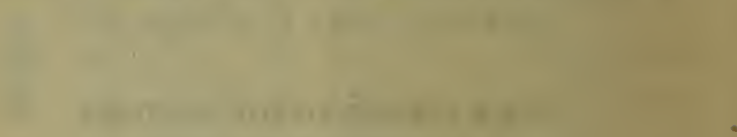

8

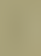
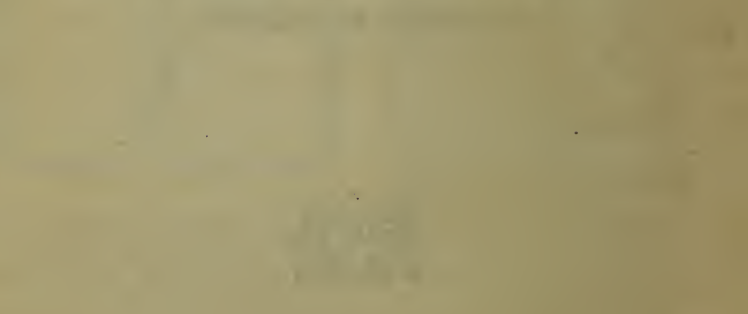

x. $6 a x+8$

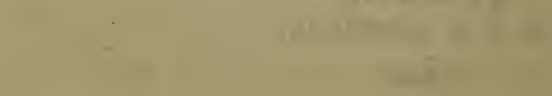




\section{OBSERVATIONŚ PRÉLIMINAIRES.}

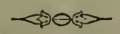

Ce travail, comme le titre l'indique, est destiné spécialement aux élèves qui suivent un cours de botanique descriptive. Les familles sont disposées suivant l'ordre proposé par. M. De Candolle, dans son livre intitulé Théorie élémentaire de Botanique, et modifié par M. A. Gray, dans son Manuel de Flore. Comme c'est aussi l'ordre que nous avons suivi dans la disposition de l'herbier de l'Université, ce Synopsis pourra aussi servir de guide à ceux qui désireraient faire une plus ample connaissance de nos plantes canadiennes.

L'Énumération des Genres sera précédée de tableaux analytiques faits sur ceux que l'on trouve au commencement de la Flore de M. A. Wood. Mais, comme ces tableaux ont été modifiés et sont spécialement fondés sur l'organisation des plantes qui se rencontrent dans les deux Canadas, ils ne sauraient servir à l'étude des plantes étrangères à notre Flore. 
Il était important de distinguer les genres indigènes de ceux qui ne le sont pas. Dans ce but, nous avons eu recours aux signes suivants :

Le signe $†$, précédant un nom de genre, indique que ce genre ne comprend que des espèces introduites ou naturalisées.

Le signe ?, qui précède certains genres, veut dire que leur indigénat est fort douteux. 


\section{TABLEAU ANALYTIQUE DES FAMILLES.}

\section{$\Leftrightarrow$}

1 Plantes portant fleurs. Plantes phanérogames........ 2

Plantes ne portant pas fleurs. Plantes cryptogames.... 5

2 Feuilles à veines ramifiées. Fleurs à type généralement quinaire, jamais ternaire .............. ExoGÈNEs. Feuilles à nervures généralement parallèles. Fleurs à type ternaire .................... EnDoG Ènes. 4

3 Ovules renfermés dans un ovaire surmonté ảe stigmate. Point de stigmate. Ovules nus. (Conifères).......... Angiospermes. 6 ......................... GYMNOSPERMEs.

4 Fleurs sans glumes; périanthe verticillé ou nul....... Aglumacées. 111 Fleurs munies de glumes; périanthe nul. Glumacées... 126

5 Axe et organes appendiculaires distincts. Acrogìnes. 127 Point d'axe'ni d'organes appendiculaires. Croissance périphérique. .................. AMPHigÈnes.

6 Corolle à pétales distincts et libres...... Polypétaless. 7 do do soudés entre eux...... Monopétales. 60 do et souvent calice, nuls............ ApÉtales. 84

\section{Polypétales.}

7 Tiges herbacées.......................... 8 Arbres, arbrisseaux et sous-arbrisseaux............. 43

8 Feuilles alternes ou toutes radicales.............. 9 Feuilles caulinaires, opposées, du moins les plus inférieures................................ 31

9 Feuilles munies de stipules................... 28 Feuilles sans stipules......................... 10

10 Fleur polyandre; étamines $17-200$, indéfinies....... 11 Fleur à étamines peu nombreuses................ 14 
11 Etamines hypogynes, insérées sur le réceptacle........ Etamines périgynes, insérées sur le calice ou sur la corolle........................ Rosace. (34).

12 Sépales 3-5. Feuilles peltées et dans l'eau. Sépales $3-9$. Feuilles ni peltées ni tubulaires......... Sepales 5, persistants. Feuilles tubulaires............

Sépales 2, caducs. Suc ordinairement coloré

SARRACENIACEN. (7). $\ldots \ldots \ldots \ldots \ldots \ldots \ldots$ Pafaverace.e. (8).

13 Pistils (ou pistil) simples et distincts, nombreux ou peu nombreux................ RanUNCULACE. (1). Pistil composé, gros, 12-30 loges. Plante aquatique. . ................... NYMPHAACE. (6).

14 Fleur bien irrégulière................... 15

Fleur régulière ou presque régulière $\ldots \ldots \ldots \ldots \ldots \ldots, 17$

15 Filets $6-8$, en 2 faisceaux................. 16 Filets 5, cohérents au sommet... BAISAMINACE E. (24).

16 Feuilles très-découpées ou divisées. . Fumariacres. (9). Feuilles simples, entières........ Polygalacee. (32).

17 Ovaire supérieur, non soudé avec le calice $\ldots \ldots \ldots \ldots .18$ Ovaire inférieur, adhérent au calice ............. 26

18 Sépales 2. Plantes à feuilles épaisses et succulentes. Portulacace. (18). Sépales 3 ou plus. Plantes vertes, munies de feuilles.. Sépales 3 ou plus. Plantes sans feuilles et non vertes. ERICACET. (52).

19 Etamines hypogynes, insérées sur le réceptacle...... 20 Etamines périgynes, insérées sur le calice ou la corolle.. 24

20 Sépales, pétales et étamines symétriques .......... 21

Sépales, pétales et étamines sans symétric. Fruit capsule, silique ou silicule...................

21 Feuilles simples $\ldots \ldots \ldots \ldots \ldots \ldots \ldots \ldots \ldots \ldots \ldots \ldots \ldots$ Feuilles composées. Suc âcre..... Oxa LIDACEN. (22).

22 Corolle persistante. Ovaire à 1 loge. Feuilles radicales. .................... DRosERACEE. (14.) Corolle caduque. Ovaire, 5-10 loges. Feuilles caulinaires...................... LINACE. (21). Corolle décidue. Ovaire à 5 loges. Feuilles caulinaires. 
23 Fleur cruciforme, régulière. Etamines tétradynames. ...................... Crucifere. (10). Fleur presque irrégulière. Etamines non tétradynames. ...................... CAPPARIDACE.. (11).

24 Etamines 5, opposées aux 5 pétales................

Etamines alternant avec les pétales, quand elles sont en même nombre que ccux-ci .. $\quad \ldots \ldots . . . . . . .$.

25 Styles $3-20$, aussi nombreux que les sépales........ ..................... Crassulacte. (39). Styles 2 , moins nombreux que les sépales........... Styles 4, moins nombreux que les sépales.

SAXIFRAGACEN. .(40). Parnassiacele. (15).

26 Fleurs à 5 parties, en ombelle simple ou composée..... Fleurs à 1, 2, 3, 4 parties, non en ombelle.

OnAGRACEe. (36).

27 Styles 2. Fruit sec; 2-partite... Umbellifere. (42). Styles $3-4$ (rarement 2). Fruit, baie ou drupe...... Araliacex. (43).

28 Fleur régulière et parfaite.................. 29

Fleur irrégulière ou monoïque................ 30

29 Etamines 00, à filets soudés en un tube entourant les 5 styles..................... MaLvace. (19). Etamines 10, unies seulement à la base et séparées des styles................... OxaLIDACE. (22). Etamines nombreuses ou peu nombreuses, libres, distincts et périgynes.............. Rosaces. (34).

30 Ovaire libre, à 3 loges, remplacé par une capsule....... VIOLACE.. (12) Ovaire libre, à 1 loge, remplacé par une gousse........ ....................... Leguminos $\pi$. (33).

31 Feuilles munies de stipules.................. 32

Feuilles sans stipules....................... 33

32 Pétales 5, à préfloraison contournée, plus grands que les sépales ................... Geraniacex. (23). Pétales 5, à préfloraison imbriquée; fleurs petites. IllecebrecB................. Caryophyllacee. (17). 
33 Fleurs bien irrégulières......... Polygalacen.. (32). Fleurs régulières ou tout au plus légèrement irrégulières...................................

34 Ovaire ou ovaires supérieurs, non soudés avec le calice.

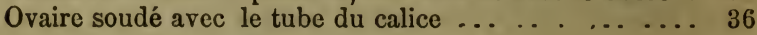

35 Ovaires multiples, distincts, simples. Ranunculacex. (1). Ovaire simple. Feuilles 2 dans le genre Podophyllum. ....... BERBERIDACE

36 Involucre à 4 folioles, blanc, inflorescence en cyme.

Involucre nul. Feuilles nombreuses et simples.......

ONAGRaces. (36). Involucre nul. Feuilles au nombre de 3, composées.... Araliace.e. (43).

37 Sépales 2, moins nombreux que les pétales. Sépales $3-5$. Style et stigmate $1 \ldots \ldots \ldots \ldots \ldots \ldots$ Sépales $3-5 . \quad$ Styles et stigmates multiples.......... 39

38 Sépales égaux, formant un calice tubuleux........... ....................... Lythraces. (35). Sépales inégaux et presque distincts.. Cistacre. (13).

39 Etamines hypogynes, insérées sur le réceptacle........ Etamines périgynes, insérées sur le calice............ 41

40 Tiges à articulations renflées. Feuilles non ponctuées.. .................... Caryophyldace.t. (17). Tiges présentant quelquefois des lignes saillantes. Feuilles ponctuées............ Hypericacex. (16).

41 Pistils (follicules) distincts, aussi nombreux que les sépales................. Crassulacex. (39). Pistils 2-5, soudés complètement ou en partie .........

42 Styles 2-5. Embryon annulaire ou semi-annulaire..... .................. CARYOPHYLLACE. (17). Styles 2. Embryon droit........ Saxirragacese. (40).

43 Feuilles opposées....................... 44 Feuilles alternes....................... 51

44 Fleurs irrégulières.... ........ Sapindacex. (31). Fleurs régulières............................ 
45 Etamines 4, aussi nombreuses que les sépales et pétales. Etamines 5, aussi nombreuses que les sépales et pétales. Etamines 6-100, plus nombreuses que les sépales et pétales.............................. 46

46 Ovaire libre ou à demi soudé, à 4 carpelles $\ldots \ldots \ldots . \quad 47$ Ovaire soudé avec le tube du calice. Onagracer. (36).

47 Etamines périgynes. Stigmates $2-4 \ldots \ldots \ldots \ldots \ldots, 49$ Etamines hypogynes. Stigmates $1-3 \ldots \ldots \ldots \ldots \ldots \ldots, 48$

48 Stigmates distincts. Feuilles non ponctuées.. . .....

Cistacee. (13). Etamines polyadelphes. Feuilles ponctuées........... HYPERICACE. (16).

49 Feuilles palmées (ou composées). Fruit, une samare... Feuilles penninerves simples. Fruit, une capsule....... ...................... Saxifragacee. (40).

50 Etamines opposées aux pétales. Tiges munies de vrilles. Vitacke. (28). Etamines alternant avec les pétales. Tige sans vrilles.. Celastracee. (30).

51 Etamines peu nombreuses et définies............ 54 Etamines nombreuses, 20 ou plus................ 52

52 Sépales 5 (rarement plus), aussi nombreux que les pétales ................................ Sépales 3 , pétales $6-9$. Feuilles stipulées .............. MagnoLIACE

53 Filets réunis, formant un tube...... MaLvacex. (19). Filets distincts, périgynes............ RosacEz. (34). Filets distincts, hypogynes.......... Truiace.e. (20).

54 Ovaire supérieur, non soudé avec le tube du calice..... 55 Ovaire soudé avec le tube du calice. Fleurs symétriques. 59

55 Corolle plus ou moins irrégulière. Fruit, une gousse... ...................... LeGUMinos Corolle régulière. Fruit, non gousse............ 56

56 Tige grimpante. Etamines 12-18. Menispermaces. (3). Tige sarmenteuse et grimpante. Etamines $5 . . . . . . .$. T....................... Cenlastrage... (30). Tige droite (arbres ou arbustes) ................... 
57 Etamines 4-5, opposées aux pétales .............. Rhamanace. (29). Etamines 6 , opposées aux pétales. Berberidacex. (4). Etamines 2-10, alternant avec les pétales, si elles sont en même nombre que ceux-ci

58 Feuilles pennées, ponctuées. Ovaire à 2 loges......... ZaNTHOXYLACE.E. (26). Feuilles presque pennées, non ponctuées. Ovaire, 1 loge $; 3$ styles.............. Anacardiace... (27). Feuilles simples, graines 4-5.... Nemopanthes....

Aquifoliacea. (5?).

59 Fleurs (en automne) à 4 parties; pétales linéaires ..... Fleurs (en juin) à 4 parties; pètales lancéolés ......................... ........................... Cornace Fleurs à 5 parties; styles $2 \ldots \ldots$ Grossulace. . (37). Fleurs à 5 partics; styles $5 \ldots \ldots \ldots$. Araliacez. (43).

\section{Monopétales.}

60 Etamines aussi nombreuses que les lobes de la corolle.. Etamines 6-12, plus nombreuses que les lobes de la corolle. ...................... ERICACEE. (52). Etamines 2-4, moins nombreuses que les lobes de la corolle...$\ldots \ldots \ldots \ldots \ldots \ldots \ldots \ldots \ldots \ldots$.

61 Fleurs sessiles sur un réceptacle commun, entourées d'un involucre.............................. Fleurs séparées et non munies d'un involucre..$\ldots \ldots .63$

62 Etamines 4, distinctes........... Dipsace.w. (48). Etamines 5, unies par les anthères... Composita. (49).

63 Calice supérieur, adhérent à l'ovaire.............. Calice inférieur, non soudé avec l'ovaire............... 67

64 Etamines unies par les anthères................. Etamines distinctes.............................

65 Fleurs régulières. Tiges munies des vrilles........... Cucurbitace......... Feurs irrégulières. Tiges sans vrilles. Lobeliace e. (50). 66 Feuilles alternes. Fleurs à 5 parties................ .................... Campanulace. (51). 
Feuilles opposées. Fleurs à 5 parties.............. CA PRIFOLIACEe. (45). Feuilles opposées. Fleurs à 4 parties. . Rubiaces. (46).

67 Plantes ì suc laiteux. Ovaires 2. Fruit, follicule..... 77 Plantes à suc aqueux. Fruit, capsule ou akène ...... 68

68 Etamines opposées aux lobes de la corolle ......... 69 Etamines alternant avec les lobes de la corolle........ 70

69 Ovaire monosperme à 5 styles.. Plumbaginacea. (55). Ovaire polysperme à 1 style. ...... Primulace. (56).

70 Arbres, arbrisseaux _.. . . . . . 71 Tige herbacée. Feuilles opposées ou toutes radicales. 73 Tige herbacée. Fcuilles alternes et caulinaires....... 75 Tige herbacée, sans feuilles Convolvulaces. (65).

71 Etamines hypogynes, libres ou très-peu adhérentes au tube de la corolle.

Etamines insérées intérieurement sur le tube de la corolle

SOLANACEe. (66).

Etamines insérées sur le sommet du tube de la corolle.. Diapensia. Polemoniacee. (64).

72 Fruit sec (capsule), polysperme ...... Ericace.e. (52). Fruit, drupe à 4-6 graines. . .... Aquifoliaces. (53).

73 Feuilles munies de stipules. Corolle à préfloraison valvaire ................... Rubiace.E. (46).

Feuilles sans stipules

74 Ovaire à 1 loge, à déhiscence circulaire. Feuilles radicales . $\quad . .6$ Plantaginaces. (54). Ovaire à 1 loge, à déhiscence bivalve. Feuilles caulinaires $\ldots$...... Gentianace 2. . (67). Ovaire à $3 \operatorname{loges,~à~déhiscence~trivalve.~Feuilles~cauli-~}$ naires Polemoniaces. (64).

75 Ovaire ì 4 parties, se séparant en 4 akènes.......... $\ldots \ldots \ldots \ldots$ BorRAGINACEA. (62). Ovaire composé, indéhiscent, à 1 loge ............. HYDROPHYLLACEN. (63). Ovaire composé, indéhiscent, à $2-3$ loges ...........

76 Corolle à préfloraison imbriquée, contournée. Enbryon plus petit que l'albumen....... POLEMONIACEE, (64). Corolle à préfloraison pliće, contournéc. Embryon plus gros que l'albumen......... ConvolvulaCE E. (65). 
Corolle à préfloraison imbriquée, mais non contournée.. C.................. SCROPHULARIACEE. (59). Corolle à prefloraison pliée ou valvaire ....

Solanacee. (66).

77 Fleurs (en ombelle) accompagnées d'une couronne à 5 lobes.................. AscLePIADACE. Fleurs (en cyme) sans couronne.... A A pocynaCEN. (68)

78 Tige herbacée. Calice adhérent à l'ovaire ... . . . . 79

Tige herbacée. Calice non soudé avec l'ovaire ... 80

Tige ligneuse. Calice non soudé avec l'ovaire. Etamines $2 \ldots \ldots \ldots \ldots$. . . . Oleace.E. (70).

79 Etamines $4 \ldots \ldots \ldots$.... Linnaca Caprifoliace. (45). Etamines $3 \ldots \ldots \ldots \ldots \ldots \ldots$ Valerianace.e. (47).

80 Plante brune, sans feuilles...... Orobanchace.. (58). Plante verte, munie de feuilles........ . . . . ... 81

81 Feuilles toutes radicales. Corolle éperonnée.......... Lentibulaces. (57). Feuilles caulinaires. Corolle sans éperon.............

82 Ovaire 4-lobé, formant 4 akènes ..... Labiata. (61). Ovaire entier, s'ouvrant en 1-4 nucules. ............

VIRBENACEN. (60). Ovaire entier, à 2 carpelles .....................

83 Corolle à préfloraison contournée.... † Acanthacex. Corolle à préfloraison imbriquée.................. .................. Scrophulariace.

\section{Apétales.}

84 Tige herbacée, à feuilles alternes ou sans feuilles ... . 85 Tige herbacée, à feuilles opposées ou verticillées ... . . 93

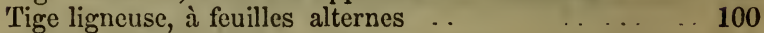
Tige ligneuse, à feuilles opposées ........ . . . 109

85 Stipules entourant la tige. Feuilles simples et entières ....................... Polygonace.t. (75). Stipules serrées, n'entourant pas la tige. Feuilles pennées................... Rosacex. (34). Stipules 0 , ou, s'il y en a, elles n'entourent pas la tige. Stipules non serrées....................... 
86 Fleur munie d'un calice régulier ou d'un involucre. . . 87

Fleur sans calice et sans involucre .............. 92

87 Calice adhérent à l'ovaire; étamines $6-12 \ldots \ldots \ldots \ldots$. ARISTOLOCHIACEE. (71)

Calice adhérent à l'ovaire; étamines $5 . \ldots \ldots \ldots \ldots \ldots$ .................. Santalacem. (79). Calice libre, non soudé avec l'ovaire ........... 88

88 Pistils entièrement distincts; étamines $00 \ldots \ldots \ldots \ldots$. ................. RanUnCUlaces. (1). Pistils soudés et formant un ovaire composé......... 89

89 Ovaire à 1 loge, renfermant une graine........... 90 Ovaire à 3 loges et 3 graines.... EupHORBIACEE. (83). Ovaire à 5 loges et 00 graines.... Crassulaces. (39). Ovaire à $6-10$ loges et $6-10$ graines................. ..................... Phytolaccaces. (72).

90 Pistil 1 ; embryon droit.......... Urticaces. (85). Pistils $2-5$; embryon courbe................ 91

91 Calice et bractées secs et scarieux ......

.................. . Amarantaces. (74). Calice (et bractées, s'il y en a,) verts.............. ...................... Chenopodiacea. (73).

92 Fleurs sur un spadice entouré d'une spathe ......... 114 Point de spadice ni de spathe..... SAURURACEe. (80).

93 Calice adhérent à l'ovaire.......... Onagrace.e. (36). Calice (ou involucre) libre................... 94 Point de calice ni d'involucre. Plante aquatique. Callitrichacex. (82).

94 Feuilles verticillées......... Ceratophyldacee. (81). Feuilles opposées $\ldots \ldots \ldots \ldots \ldots \ldots \ldots \ldots \ldots \ldots \ldots$

95 Herbes à suc laiteux. Fruit contenant 3 graines..... EUPHORBIACE. (83).

Herbes à suc aqueux $\ldots \ldots \ldots \ldots \ldots$
96 Feuilles munies de stipules, feulles lobées ou composées. Feuilles munies de stipules, simples et entières....

Caryophyllacke. (17). Feuilles sans stipules.

97 Etamines 00, bien plus nombreuses que les sépales..... Ranunculace.e. (1). 
Etamines en nombre égal aux sépales ou moins nombreuses............................

98 Calice et bractées secs et scarieux

Amarantacen. (74). Calice non scarieux et sans bractées.....

99 Etamines alternant avec les sépales. Fleurs parfaites .. .................. Primulace. (56). Etamines opposées aux sépales. Fleurs parfaites......

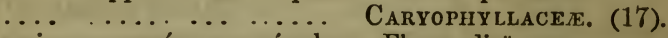
Etamines opposées aux sépales. Fleurs dioïques....... $\ldots \ldots \ldots \ldots \ldots \ldots$ URTICACE. (85).

100 Fleurs presque parfaites, avec calice, jamais disposées en chaton ............................. 101

Fleurs incomplètes, $\delta$ en chaton $\ldots \ldots \ldots . . . \ldots 105$

Fleurs incomplètes, les $\delta$ et $q$ en chaton ......... 106

101 Etamines alternant avec les sépales et en nombre égal... 102 Etamines opposées aux sépales et plus nombreuses. 103

102 Feuilles dentées en scie.......... Rhamnacks. (29). Feuilles entières couvertes d'écailles discoïdes ......... ..................... Elegnaces. (78). Feuilles entières et toujours vertes EMPETRACEN. (84).

103 Sépales 3 ; étamines $6 \ldots \ldots$..... Aristolochiac 2. . (71). Sépales 4 ; étamines $8 . .$. Thymelacex. (77). Sépales 6 ; étamines 9 , sur 3 rangs... LAURACE $E . ~(76)$. Sépales 5-9; étamines 5-9, sur 1 rang . . . . . 104

104 Feuilles composées et ponctuées. ZanthoxyLACE. (26). Feuilles simples. Calice adhérent à l'ovaire.......

Santalaceis. (79). Feuilles simples. Calice libre, non sondé avec l'ovaire.. $\ldots \ldots \ldots . \ldots . . . . \quad$ URTICACE.E. (85).

105 Feuilles simples. Noix munies de cupules. Feuilles composées. Noix sans cupules

Cupulifere. (88). $\ldots \ldots \ldots$ JUG I.ANDACE

106 Plantes à suc laiteux. Fruit charnu.. Unticaces. (85). Plantes à suc aqueux. Fruit sec..

107 Chatons globuleux, pendants...... Platanace.r. (86). Chatons cylindriques ou ovoïdes 
108 Ovaire à $1 \operatorname{loge}$; ovule 1 et fruit à une graine........... MYRICACEN. (89). Ovaire à 2 loges; ovules 2 et fruit à 1 seule graine. ... Ovaire à 00 orules, et fruit à 00 graines ... ...... Salicace 2. (91).

109 Calice à $3-4$ parties ... . . . . . . . . . . . . 110 Calice à 5 parties. Fruit, une double samare.......... SaPINDACEK. (31).

110 Etamines 2. Fruit, 1 samare......... Oleacere. (70). Etamines 8. Feuilles couvertes d'écailles discoïdes..... Eltanace . (78).

\section{Endogènes aglumacées.}

111 Tige ligneuse et grimpante. Fleurs en ombelle....... 122 .................... Smilacem. (102).

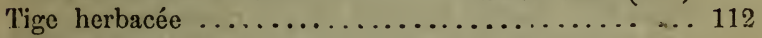

112 Périanthe complet, à 6 parties (ou à 4 ). Jamais sur un spadice ........................... 116 Périanthe complet, en tête globuleusc........

Eriocaulonace. (107). Périanthe 0 ou incomplet, presque toujours sur un spa-

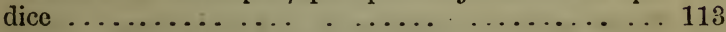

113 Plantes terrestres................ . .. 114 Plantes aquatiques...................... 115

114 Axe central (spadice) muni d'une spathe. Aracex. (93). Axe central sans spathe. ........ TyPHACEæ. (94).

115 Plante flottante à la surface de l'eau.. LEMnaces. (\$5). Plante submergée............... NAtadace.. (96).

116 Périanthe adhérent à l'ovaire (dans les fleurs parfaites). 117 Périanthe non adhérent à l'ovaire. ............. 120

117 Fleurs complètes; plantes terrestres . ........... 118 Fleurs monoïques ou dioïques ; plantes aquatiques. ...... HYDROCHARIDACE.E. (98).

118 Fleurs irrégulières $\ldots \ldots \ldots \ldots \ldots \ldots \ldots \ldots . . \ldots \ldots$

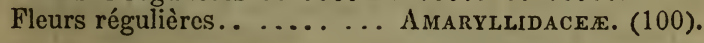

119 Etamines 1-3, adhérent au pistil... ORCHIDACEe. (99). Etamines 3 et libres.............. IrIdaCEe. (101). 
120 Pétales et sépales de différente couleur........... 125

Pétales et sépales de même couleur .............. 121

121 Styles et stigmates 3 , distincts. . . . . . . . . . . 122

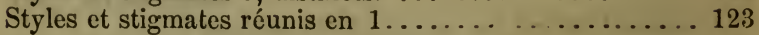

122 Feuilles à nervures ramifiées, pétiolées. SmrLace.e. (102). Feuilles à nervures parallèles, capsule polysperme...... Melanthacez. (104). Feuilles à nervures parallèles, fruit s'ouvrant en 2 par. ties à $1-2$ graines............ Alrsmace. . (97).

123 Périanthe coloré.

Périanthe sec, vert ou scarieux...... JunCACEE. (105).

124 Fleurs régulières, hexandres ....... LrLIACEe. (103). Fleurs irrégulières, triandres. Plante aquatique....... Pontederiacex. (106).

125 Styles et stigmates 3 . Feuilles à veines ramifiées...... ................ Trilliacece. Smrlacex. (102). Styles 00 . Feuilles à veines un peu ramifiées ........ ...................... Alismace... (97).

\section{Endogènes glumacées.}

126 Fleurs solitaires chacune à l'aisselle d'une bractée; feuilles à gaîne non fendue...... Cyperacex. (108). Fleurs entourées de 2 bractées; feuilles à gaîne fendue.. . Graminex. (109).

\section{Acotylédonnées ou Acrogènes.}

127 Fructifications sur la face inférieure des feuilles........ (Fougères.) Filices. (111). Fructifications disposées en épi au sommet de la tige ou des rameaux............... Equisetace. (110). Fructifications à l'aisselle des feuilles.

LYCOPODIACE. (112).

Fructifications à la base des feuilles ou des branches. Plantes aquatiques......... Hydropterides. (113). 


\section{ÉNUMERATION}

\section{DES \\ GENRES DE PLANTES \\ DE LA FLORE DU CANADA.}

\section{DICOTYLÉ DONNÉES ou EXOGÈNES.}

Embryon à deux cotylédons opposés, tiges présentant des faisceaux fibro-vasculaires formant un cylindre autour d'une moelle centrale, séparable en une zone ligneuse et une zone corticale, et s'accroissant par des couches concentriques.

\section{POLYPÉTALES.}

\section{RANUNCULACEE.}

TRIBU I. CLEMATIDEA.

1. Atragene, $L$.

2. Clematis, $\boldsymbol{L}$.

TRIBU II. ANEMONE王.

3. Anemone, $L$.

4. Hepatica, Dill.

5. Thalictrum, Tourn.

TRIBU III. RANUNCULEE.

6. Ranunculus, $L$. 
TRIBU IV. HELLEBORINE E.

7. Caltha, $L$.

8. Coptis, Salisb.

9. Aquilegia, Tourn.

10. Hydrastis, $L$. *

TRIBU V. CIMICIFUGE E.

11. Actæa, $L$.

2. MAGNOLIACE $Æ$.

? 1. Magnolia, $L$.

2. Liriodendron, $\boldsymbol{L}$.

3. MENISPERMACE E.

Menispermum, $\boldsymbol{L}$.

4. BERBERIDACE $\not E$.

1. Caulophyllum, Michx.

2. Jeffersonia, Barton. .

3. Podophyllum, $L$.

5. САВOMBACE $\not E$.

Brasenia, Schreber.

6. NYMPHÆACE $Æ$.

1. Nymphæa, Tourn.

2. Nuphar, Smith.

7. SARRACENIACEÆE.

Sarracenia, Tourn.

* L'Hydrastis Canadensis est une plante commune dans le township de Williams, près de London, Haut-Canada. 
8. PAPAVERACEE.

1. Sanguinaria, Dill.

† 2. Chelidonium, $L$.

9. FUMARIACE $Æ$.

1. Adlumia, $R a f$.

2. Dicentra, Bork.

3. Corydalis, Vent.

$\dagger$ 4. Fumaria, $L$.

10. CRUCIFER E.

SOUS-FAMILLE I. SILIQUOSE.

TRIBU I. ARABIDEE.

1. Nasturtium, R. Br.

2. Iodanthus, Torr. \& Gray.

3. Dentaria, $L$.

4. Cardamine, $L$.

5. Arabis, $L$.

6. Turritis, Dill.

7. Barbarea, R. Br.

TRIBU II. SISYMBRIEA.

7. Erysimum, $L$.

8. Sisymbrium, $L$.

TRIBU III. BRASSICEAE.

$\dagger$ 9. Sinapis, Tourn.

SOUS-FAMILLE II. SILICULOSA.

TRIBU IV. ALYSSINE $Æ$.

10. Draba, $L$.

(1. Nasturtium, R. Br.)

TRIBU V. CAMELINE瓜.

† 11. Camelina, Crantz. 


\section{ÉNUMÉRATION DES}

TRIBU VI. LEPIDINEA.

12. Lepidium, $L$.

† 13. Capsella, Vent. SOUS-FAMILLE III. LOMENTACEA.

TRIBU VII. CAKILINEA.

14. Cakile, Tourn.

11. CAPPARIDACEAE.

Polanisia, Raf.

12. VIOLACE无.

Viola, $\boldsymbol{L}$.

13. CISTACE $A$.

1. Helianthemum, Tourn.

2. Hudsonia, $L$.

3. Lechea, $L$.

14. DROSERACEÆ.

Drosera, $L$.

15. PARNASSIACE E.

Parnassia, Tourn.

16. HYPERICACE $Æ$.

1. Hypericum, $L$.

2. Elodea, Pursh.

17. CARYOPHYLLACEÆ.

SoUs-FAMILLE I. SILENEE.

1. Silene, $L$.

2. Agrostemma, $L$. 
SOUS-FAMILLE II. ALSINEÆ.

3. Arenaria, $L$.

4. Mœhringia, $L$.

5. Stellaria, $\boldsymbol{L}$.

6. Cerastium, $L$.

7. Sagina, $L$.

SOUS-FAMILLE III. ILLECEBREÆ.

8. Spergularia, Pers.

† 9. Spergula, $\boldsymbol{L}$.

SoUs-Famile IV. MOLLUGINEÆ.

Mollugo, $L$.

18. PORTULACACE E.

1. Claytonia, $L$.

† 2. Portulaca, Tourn.

19. MALVACE

$\dagger$ Malva, $\boldsymbol{L}$.

20. TILIACE $\$$.

Tilia, $L$.

21. LINACE E.

Linum, $L$.

22. OXALIDACE $\nexists$.

Oxalis, $L$.

23. GERANIACEÆ.

Geranium, $\boldsymbol{L}$. 
GNUMÉRATION DES

24. BALSAMINACE $Æ$.

Impatiens, $L$.

25. LIMNANTHACE $A$.

Flœrkea, Willd.

26. ZANTHOXYLACE Æ.

Zanthoxylum, Colden.

27. ANACARDIACE $\not$.

Rhus, $\boldsymbol{L}$.

28. VITACE $Æ$.

1. Vitis, Tourn.

2. Ampelopsis, Michx.

29. RHAMNACE AE.

1. Rhamnus, Tourn.

2. Ceanothus, $L$.

30. CELASTRACE $A$.

1. Celastrus, $L$.

2. Euonymus, Tourn.

31. SAPINDACE $A$.

SOUS-FAMILI. I. STAPHYLEACEA.

1. Staphylea, $L$.

SOUS-FAMILLE II. SAPINDACEA.

† 2. Asculus, $L$.

SOUS-FAMILLE III. ACERINEÆE.

3. Acer, Tourn. 
GENRES DE PLANTES.

32. POLYGALACE $Æ$.

Polygala, Tourn.

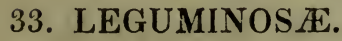

SOUS-FAMILLE I. PAPILIONACEA.

TRIBU I. LOTEE.

Sous-Tribu I. GENISTEA.

1. Lupinus, Tourn.

Sous-Tribu II. TRIFOLIE

2. Trifolium, $L$.

† 3. Melilotus, Tourn.

Sous-Tribu IIJ. GALEGEX.

† 4. Robinia, $L$.

Sous-Tribu IV. ASTRAGalE

5. Astragalus, $\boldsymbol{L}$.

6. Oxytropis, D. C.

TRIBU II. HEDYSARE压.

7. Hedysarum, Tourn.

8. Desmodium, D. C.

9. Lespedeza, Michx.

TRIBU III. VICIEA.

10. Vicia, Tourn.

11. Lathyrus, $L$.

TRIBU IV. PHASEOLEF.

12. Phaseolus, $L$.

13. Apios, Boerh.

15. Amphicarpæa, Ell.

TRIBE V. PODALYKIE E.

15. Baptisia, 'Vent. 
SOUS-FAMILLE II. CAESALPINIE尼.

† 16. Gymnocladus, Lam.

+ 17. Gleditschia, $L$.

34. ROSACE $A$.

SOUS-FAMILLE I. AMYGDALEA.

1. Prunus, $L$. SOUS-FAMILLE II. ROSACE正.

TRIBU I. SPIR压E.

2. Spiræa, $L$.

3. Gillenia, Mœnch.

TRIBU II. DRYADE $\&$.

Sous-Tribu I. SANGUISORBEX.

4. Agrimonia, Tourn.

5. Sanguisorba, $\boldsymbol{L}$.

Sous-Tribu II. EUDRYADEE.

? 6. Dryas, $L$.

7. Geum, $\boldsymbol{L}$.

8. Waldsteinia, Willd.

Sous-Tribu III. FRAGARIEN.

9. Potentilla, $\boldsymbol{L}$.

10. Fragaria, Tourn.

Sous-Tribu iv. DALIBARDEX.

11. Dalibarda, $L$.

12. Rubus, $L$.

TRIBU III. ROSEE.

13. Rosa, Tourn.

SOUS-FAMILLE III. POMEE.

14. Cratægus, $L$.

15. Pyrus, $L$.

16. Amelanchier, Medic. 
35. LYTHRACE $Æ$.

1. Lythrum, $L$.

2. Nesæa, Commerson.

36. ONAGRACE压.

SOUS-FAMILLE I. ONAGRACEE.

1. Epilobium, $L$.

2. Enothera, $L$.

3. Gaura, $L$.

4. Ludwigia, $L$.

5. Circæa, Tourn.

SOUS-FAMILLE II. HALORAGEA.

6. Proserpinaca, $L$.

7. Myriophyllum, Vaill.

8. Hippuris, $L$.

37. GROSSULACEA.

Ribes, $L$.

38. CUCURBITACEAE.

1. Sicyos, $L$.

2. Echinocystis, Torr. \& Gray.

39. CRASSULACEÆ.

1. Sedum, $L$.

2. Penthorum, Gronov.

40. SAXIFRAGACEA:

SOUS-FAMILLE I. SAXIFRAGACEA.

1. Saxifraga, $L$.

2. Heuchera, $L$. 
3. Mitella, Tourn.

4. Tiarella, $L$.

5. Chrysosplenium, Tourn.

41. HAMAMELACE E.

Hamamelis, $\boldsymbol{L}$.

42. UMBELLIFERA.

SOUS-FAMILLE I. ORTHOSPERMA.

1. Hydrocotyle, Tourn.

2. Sanicula, Tourn.

3. Heracleum, $L$.

$\dagger$ 4. Pastinaca, Tourn.

5. Archangelica, Hoffm.

6. Conioselinum, Fisher.

7. Ligusticum, $\boldsymbol{L}$.

8. Thaspium, Nutt.

9. Zizia, D. $C$.

10. Cicuta, $L$.

11. Sium, $L$.

12. Cryptotænia, D. C.

SOUS-FAMILLE II. CAMPYLOSPERMA.

13. Osmorrhiza, Raf.

$\dagger$ 14. Conium, $L$.

SOUS-FAMILLE III. CEELOSPERMA.

15. Erigenia, Nutt.

43. ARALIACE E.

Aralia, Tourn.

44. CORNACEE.

Cornus, Tourn. 


\section{MONOPÉTALES.}

\section{CAPRIFOLIACE $Æ$.}

TRIBU I. LONICEREA.

1. Linnæa, Gronov.

2. Symphoricarpus, Dill.

3. Lonicera, $L$.

4. Diervilla, Tourn.

5. 'Triosteum, $L$.

TRIBU II. SAMBUCEA.

6. Sambucus, Tourn.

7. Viburnum, $L$.

46. RUBIACEA.

SOUS-FAMILLE I. STELLAT A.

1. Galium, $L$.

SOUS-FA Mille II. CINCHONEAE.

2. Cephalanthus, $L$.

3. Mitchella, $\boldsymbol{L}$.

4. Oldenlandia, $L$.

47. VALERIANACEAE.

Valeriana, Tourn.

48. DIPSACE

† Dipsacus, Tourn.

49. COMPOSITAE.

SOUS-FA MILLE I. TUBULIFLOR A.

TRIBU I. VERNONIACE王.

1. Vernonia, Schreb. 
TRIBU II. EUPATORIACEN.

Sous-Tribu I. EUPATORIE E.

2. Liatris, Schreb.

3. Eupatorium, Tourn.

SuIs-Tribu II. TUSSILAGINEA.

4. Nardosmia, Cass. TRISU III. ASTEROIDEEE.

Sous-Tribu I. ASTERINEE.

5. Sericocarpus, Necs.

6. Aster, $L$.

7. Erigeron, $L$.

8. Diplopappus, Cass.

9. Boltonia, L'Hér.

10. Solidago, $L$.

Sous-Tribu II. INULEE.

$\dagger$ 11. Inula, $L$.

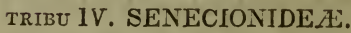

Sous-Tribu I. AIELAMPODINEF.

12. Polymnia, $L$.

13. Ambrosia, Tourn.

14. Xanthium, Tourn.

Sous-Tribu II. HELIantue $\pi$.

15. Rudbeckia, $L$.

16. Helianthus, $L$.

17. Coreopsis, $L$.

18. Bidens, $L$.

Sous-Tribu IIr. HELENIE.E.

19. Helenium, $L$.

Sous-Tribu iv, ANTHEMIDEE.

† 20. Maruta, Cass.

21. Achillea, $L$.

$\dagger$ 22. Leucanthemum, Tourn.

23. Tanacetum, $L$.

24. Artemisia, $L$. 
GENRES DE PLANTES.

Sous-Tribu v. GNAPHALINEX.

25. Gnaphalium, $L$.

26. Antennaria, Gærtn.

Sous-Tribu vi. SENECIONEXE.

27. Erechthites, Raf.

28. Senecio, $L$.

29. Arnica, $L$.

TRIBU V. CYNARE压.

$\dagger$ 30. Centaurea, $L$.

31. Cirsium, Tourn.

† 32. Lappa, Tourn.

SOUS-FAMILLE II. LIGULIFLURÆ.

† 33. Lampsana, Tourn.

† 34. Cichorium, Tourn.

35. Hieracium, Tourn.

36. Nabalus, Cass.

37. Taraxacum, Haller.

38. Lactuca, 'Tourn.

39. Mulgedium, Cass.

$\dagger$ 40. Sonchus, $L$.

50. LOBELIACE 2 .

Lobelia, $L$.

51. CAMPANULACE $Æ$.

1. Campanula, Tourn.

2. Specularia, Heist.

52. ERICACE $Æ$.

SOUS-FAMILLE I. VACCINIEÆ.

1. Gaylussacia, H. B. K.

2. Vaccinium, $L$.

3. Chiogenes; Salisb. 
SOUS-FAMILLE II. ERICINEÆ.

TRIBU 1. AKBUTE死.

4. Arctostaphylos, Adams.

TRIBU II. ANDROMEDE $\approx$.

5. Epigæa, $\boldsymbol{L}$.

6. Gaultiera, Brunet. (Gaultheria, Kalm; Gautiera, Endl.; Gualtheria, R. Br.)

7. Cassandra, Don.

8. Cassiope, Don.

9. Andromeda, $L$.

TRIBU III. RHODORE $\approx$.

10. Kalmia, $L$.

11. Azalea, $L$.

12. Rhododendron, $L$.

13. Rhodora, Duham.

14. Ledum, $L$.

15. Loiseleuria, Desv.

SOUS-FAMILLE III. PYROLEÆ.

16. Pyrola, $L$.

17. Moneses, Salisb.

18. Chimaphila, Pursh.

SOUS-FAMILLE IV. MONOTROPEA.

19. Pterospora, Nult.

20. Monotropa, $L$.

53. AQUIFOLIACE $Æ$.

1. Nemopanthes, Raf.

2. Ilex, $L$.

54. PLANTAGINACE E.

Plantago, $L$. 
GENRES DE PLANTES.

55. PLUMBAGINACEE.

? Statice, Tourn.

56. PRIMULACE压.

TRIBU I. PRIMULE瓜.

1. Primula, $L$.

2. Trientalis, $\boldsymbol{L}$.

3. Lysimachia, $L$.

4. Naumburgia, Monch.

5. Glaux, $L$.

TRIBU II. SAMOLEZE.

6. Samolus, $L$.

57. LENTIBULACEA.

1. Utricularia, $L$.

2. Pinguicula, $L$.

58. OROBANCHACEÆ.

1. Epiphegus, Nutt.

2. Conopholis, Wallr.

3. Aphyllon, Mitchell.

59. SCROPHULARIACE $\AA$.

§1. ANTIRRHINIDEÆ.

TRIBU I. VERBASCE压.

† 1. Verbascum, $L$.

TRIBU II. ANTIRRHINEE.

2. Linaria, Tourn. TRIBU III. CHELONEX.

3. Scrophularia, !Tourn.

4. Chelone, Tourn.

5. Penstemon, Mitchell, 


\section{ÉNUMÉRATION DES}

TRIBU IV. GRATIOLEAE.

6. Mimulus, $L$.

7. Gratiola, $L$.

8. Ilysanthes, Raf.

\section{§ 2. RHINANTHIDE $\mathrm{E}$.}

TRIEU V. SIBTHORPIEA.

9. Limosella, $L$.

TRIBU Vl. VERONICE A.

10. Veronica, $L$.

TRIBU VII. GERARDIE压.

11. Gerardia, $L$. TRIBU VIII. EUPHRASIE $E$.

12. Castilleia, Mutis.

13. Euphrasia, Tourn.

14. Rhinanthus, Tourn.

15. Pedicularis, Tourn.

16. Melampyrum, Tourn.

60. VERBENACEA.

1. Verbena, $\boldsymbol{L}$.

2. Phryma, $L$.

61. LABIATAE.

TRIBU I. AJUGOIDEAE.

1. Teucrium, $L$.

? 2. Trichostema, $L$.

TRIBU II. SATUREIE Æ.

3. Mentha, $L$.

4. Lycopus, $L$.

5. Cunila, $L$.

6. Pycnanthemum, Michx.

7. Calamintha, Monch. 
GENRES DE PLANTES.

8. Hedeoma, Pers.

9. Collinsonia, $\boldsymbol{L}$.

TRIBU III. MONARDE E.

10. Monarda, $L$.

TRIEU IV. NEPETE $A$.

11. Lophanthus, Benth.

$\dagger$ 12. Nepeta; $L$.

13. Dracocephalum, $L$.

TRIBU V. STACHYDEA.

14. Physostegia, Benth.

15. Brunella, Tourn.

16. Scutellaria, $L$.

+ 17. Marrubium, $L$.

+ 18. Galeopsis, $L$.

19. Stachys, $L$.

+ 20. Leonurus, $L$.

† 21. Lamium, $L$.

\section{BORRAGINACEA.}

TRIBU I. BORRAGEA.

† 1. Echium, Tourn.

† 2. Lycopsis, $\boldsymbol{L}$.

† 3. Symphytum, Tourn.

4. Onosmodium, Michx.

5. Lithospermum, Tourn.

6. Mertensia, Roth.

7. Myosotis, $L$.

† 8. Echinospermum, Swarlz.

9. Cynoglossum, Tourn.

63. HYDROPHYLLACE 压:

Hydrophyllum, $L$. 
64. POLEMONIACEA:

1. Phlox, $L$.

2. Diapensia, $L$.

65. CONVOLVULACE E.

tRIBU I. CONVOLVULE

1. Calystegia, $R$. Br. TRIBU II. CUSCUTINEA.

2. Cuscuta. Tourn. 66. SOLANACE $Æ$.

† 1. Solanum, $L$.

2. Physalis, $L$.

† 3. Hyoscyamus, Tourn.

† 4. Datura, $L$.

67. GENTIANACEAE.

TRIBU I. GENTIANE瓜.

1. Sabbatia, Adams.

2. Frasera, Walt.

3. Halenia, Borkh.

4. Gentiana, $L$.

5. Pleurogyne, Griseb.

TRIBU II. MENYANTHEA. .

6. Menyanthes, Tourn.

7. Limnanthemum, Gmelin.

68. APOCYNACE $\mathrm{E}$.

Apocynum, Tourn.

69. ASCLEPIADACEÆ.

1. Asclepias, $L$.

2. Acerates, Ell. 
ÉNUMERATION DES

70. OLEACEA.

TRIBU I. FRAXINEA.

Fraxinus, Tourn.

\section{APETALES.}

71. ARISTOLOCHIACEA.

Asarum, Tourn.

72. PHYTOLACCACEA.

Phytolacca, Tourn.

73. CHENOPODIACEAE.

$\S 1$. CYCLOLOBEA.

TRIBU I. CHENOPODIEE.

† 1. Chenopodium, $L$.

2. Blitum, Tourn.

IRIBU II. SPINACIEE.

3. Atriplex, Tourn.

TRIBU III. SALICORNIEE.

4. Salicornia, Tourn.

§ 2. SPIROBOLE王.

TRIBU IV. SALSOLEE.

5. Salsola, $L$.

74. AMARANTACEA.

† 1. Amarantus, Tourn.

2. Montelia, Moquin. 
75. POLYGONACEA.

1. Polygonum, $L$.

$\dagger$ 2. Fagopyrum, Tourn.

3. Rumex, $L$.

76. LAURACEA.

1. Sassafras, Nees.

2. Benzoin, Nees.

77. THYMELEACE Æ.

Dirca, $L$.

78. ELÆAGNACE

1. Shepherdia, Nutt.

2. Elæagnus, $L$.

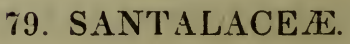

Commandra, Nutt.

80. SAURURACE E.

? Saururus, $L$.

81. CERATOPHYLLACEA.

Ceratophyllum, $L$.

82. CALLITRICHACEÆ.

Callitriche, $L$.

83. EUPHORBIACEA.

1. Euphorbia, $L$.

2. Acalypha, $L$.

84. EMPETRACEA.

Empetrum, Tourn. 
85. URTICACE E.

- SOUS-FAMILLE I. ULMACEA.

1. Uimus, $L$.

2. Celtis, Tourn.

SOUS-FAMILLE II. URTICEß.

3. Urtica, Tourn.

4. Laportea, Gaudich.

5. Pilea. Lindl.

6. Bœhmeria, Jacq.

SOUS-FAMILLE III. CANNABINEE.

$\dagger$ 7. Cannabis, Tourn.

8. Humulus, $L$.

86. PLATANACE $Æ$.

Platanus, $L$.

87. JUGLANDACEA.

1. Juglans, $L$.

2. Carya, Nult.

88. CUPULIFER E.

1. Quercus, $L$.

2. Castanea, Tourn.

3. Fagus, Tourn.

4. Corylus, Tourn.

5. Carpinus, $L$.

6. Ostrya, Micheli.

89. MYRICACEAE.

1. Myrica, $L$.

2. Comptonia, Solander. 
90. BETULACE

1. Betula, Tourn.

2. Alnus, Tourn.

91. SALICACEA.

1. Salix, Tourn.

2. Populus, Tourn.

92. CONIFER $Æ$.

SOUS-FAMILLE I. ABIETINEA.

1. Pinus, Tourn.

2. Abies, Tourn.

3. Picea, Link.

4. Larix, Tourn.

SOUS-FAMILLE II. CUPRESSINEA.

5. Thuja, Tourn.

6. Juniperus, $L$.

SOUS-FAMILLE III. TAXINEE.

7. Taxus, Tourn.

\section{MONOCOTYLEDONNEES ou ENDOG TENES.}

Embryon à un seul cotylédon.-Tiges composées de faisceaux fibro-vasculaires, épars dans la masse du tissu cellulaire, ne s'accroissant pas par zones concentriques.

\section{AGLUMACE.E.}

93. ARACEA.

1. Arisæma, Marlius. 
GENRES DE PIANTES.

2. Calla, $L$.

3. Symplocarpus, Salisb.

4. Acorus, $L$.

94. TYPHACEÆ.

1. Typha, Tourn.

2. Sparganium, Tourn.

95. LEMNACE E.

Lemna, $L$.

96. NAIADACEA.

1. Naias, $L$.

2. Zannichella, Micheli.

3. Potamogeton, Tourn.

97. ALISMACEA.

SOUS-FAMILlE I. JUNCAGINEÆ.

1. Triglochin, $L$.

2. Scheuchzeria, $L$.

SOUS-FAMILLE II. ALISMEÆ.

3. Alisma, $L$.

4. Sagittaria, $L$.

98. HYDROCHARID ACE $A$.

1. Limnobium, Richard.

2. Anacharis, Richard.

3. Valisneria, Micheli.

99. ORCHIDACE $\nexists$.

TRIBU I. OPHRYDE ÆE.

1. Orchis, $L$.

2. Gymnadenia, R. Br.

3. Platanthera, Richard. 
TRIB十 II. NEOTTIEA.

4. Goodyera, $R$. Br:

5. Spiranthes, Richard.

6. Listera, $R$. $B r$.

TRIBU III. ARETHUSER, \&c.

7. Arethusa, Groncv.

8. Pogonia, Juss.

9. Calopogon, R. Br.

10. Calypso, Salisb.

11. Microstylis, Nutt.

12. Liparis, Richard.

13. Corallorhiza, Haller.

14. Amplectrum, Nutı.

TRIBU IV. CYPRIPEDIEA.

15. Cypripedium, $L$.

100. AMARYLLIDACE正.

? Hypoxys, $L$.

101. IRIDACE $\mathbb{E}$.

1. Iris, $L$.

2. Sisyrinchium, $L$.

102. SMILACE $A$.

SOZS-FAMILLE I. EUSMILACEÆ.

1. Smilax, Tourn.

SOUS-FAMILLE II. TRILLIACE $Æ$.

2. Trillium, $L$.

3. Medeola, Gronov:

103. LILIACE $\nexists$.

TRIBU I. ASPARAGE E.

1. Polygonatum, Tourn. 
GENRES DE PLANTES.

2. Smilacina, Desf.

3. Clintonia, $R a f$.

TRIBU II. ASPHODELEA.

4. Allium, $L$.

TRIBU III. TULIPACE.A.

5. Lilium, $L$.

6. Erythronium, $L$.

104. MELANTHACE $\nexists$.

SOUS-FANILLE I. UVULARIEA.

1. Uvularia, $L$.

2. Streptopus, Michx.

SOUS-FAMILLE II. MELANTHIEZE.

3. Melanthium, Gronov.

4. Zygadenus, Michx.

5. Veratrum, Tourn.

6. Chamælirium, Willd.

7. Tofieldia, Hudson.

105. JUNCACEE.

1. Luzula, D. $C$.

2. Juncus, $L$.

106. PONTEDERIACEÆ.

1. Pontederia, $L$.

2. Schollera, Schreber.

107. ERIOCAULONACE E.

Eriocaulon, $L$. 


\section{GLUMACE涩.}

108. CYPERACE $R$.

TRIBU I. CYPEREA.

1. Cyperus, $L$.

2. Dulichium, Richard.

TRIBU II. SCIRPEÆ.

3. Eleocharis, R. Br.

4. Scirpus, $L$.

5. Eriophorum, $L$.

TRIBU III. RHYNCHOSPOREA.

6. Rhynchospora, Vahl.

TRIBU IV. CARICEX.

7. Carex,$L$.

109. GRAMINE无.

TRIBU I. POACEA.

Sous-Tribu I. ORYZEAE.

1. Leersia, Solander.

2. Zizania, Gronov.

Sous-Tribu II. AGROSTIDE E.

3. Alopecurus, $L$.

4. Phleum, $L$.

5. Agrostis, $L$.

6. Cinna, $L$.

7. Muhlenbergia, Schreber.

S. Brachyelytrum, Beauv.

9. Calamagrostis, Adams.

10. Oryzopsis, Michx.

11. Stipa, $L$.

Sous-Tribu IIr. CHLORIDEA.

12. Spartina, Schreber. 
Sous-Tribu IV. FESTUCEA.

13. Dupontia, $R . B r$.

14. Dactylis, $L$.

15. Eatonia, Raf.

16. Glyceria, R. Br.

17. Poa, $L$.

18. Festuca, $L$.

19. Bromus, $L$.

20. Phragmites, ${ }^{\text {Trin }}$.

Sous-Tribu v. HORDEINE

† 21. Lolium, $L$.

22. Triticum, $L$.

23. Hordeum, $L$.

24. Elymus, $L$.

25. Gymnostichum, Schreb.

Sous-Tribu VI. AVENE E..

26. Aira, $L$.

27. Trisetum, Persoon.

28. Avena, $L$.

† 29. Holcus, $L$.

TRIBU II. PHALARIDEAE.

Sous-Tribu 1. ANThoXanthez.

30. Hierocloa, Gmelin.

31. Anthoxanthum, $L$.

Sous-Tribu II. Phalaride

32. Phalaris, $L$.

TRIBU II1. PANICEA.

33. Milium, $L$.

34. Panicum, $L$.

† 35. Setaria, Beauv.

36. Andropogon, $L$.

37. Sorghum, Pers. 


\section{ACOTYLÉ DONVÉES ou ACROGL̀NES.}

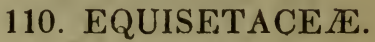

Equisetum, $L$.

111. FILICES. Fougères.

SOUS-FAMILLE I. POLYPODINEA.

TRIBU I. POLYPODIEA.

1. Polypodium, $L$.

2. Struthiopteris, Willd.

TRIBU II. PTERIDE $£$.

3. Allosurus, Bernhardi.

4. Pteris, $L$.

5. Adiantum, $L$.

TRIBU III. ASPLENIE

6. Camptosorus, Link.

7. Asplenium, $L$.

TRIBU IV. DICKSONIE

8. Dicksonia, L'Hér.

TRIBU V. WOODSIEE.

9. Woodsia, R. Br.

TRIBU VI. ASPIDIE

10. Cystopteris, Bernhardi.

11. Aspidium, Swartz.

12. Onoclea, $L$.

SOUS-FAMILLE Il. OSMUNDINEE.

13. Osmunda, $L$.

SOUS-FAMILLE III. OPHIOGLOSSEA.

14. Botrychium, Swartz. 
GENRES DE PLANTES.

112. LYCOPODIACE E.

1. Lycopodium, $L$.

2. Selaginella, Beauv.

\section{HYDROPTERIDES.}

1. Isoetes, $L$.

2. Azolla, Lam. 



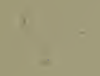



